\title{
POTENTIAL MECHANISMS OF CANCER PREVENTION BY WEIGHT CONTROL
}

\author{
Yu Jiang \\ Department of Human Nutrition, Kansas State University, \\ Manhattan, KS 66506, USA \\ yjiang@ksu.edu \\ Weiqun Wang* \\ Department of Human Nutrition, Kansas State University, \\ Manhattan, KS 66506, USA \\ wwang@ksu.edu
}

*Correspondence at: Phone: 1-785-532-0153; Fax: 1-785-532-3132; Email: wwang@ksu.edu

\author{
Received July 17, 2008
}

Revised July 25, 2008

\begin{abstract}
Weight control via dietary caloric restriction and/or physical activity has been demonstrated in animal models for cancer prevention. However, the underlying mechanisms are not fully understood. Body weight loss due to negative energy balance significantly reduces some metabolic growth factors and endocrinal hormones such as IGF-1, leptin, adiponectin, and glucocorticoids, etc., that may be associated with anti-cancer mechanisms. In this review, we summarized the recent studies related to weight control and growth factors. The potential molecular targets focused on those growth factors- and hormones-dependent cellular signaling pathways are further discussed. It appears that multiple factors and multiple signaling cascades, especially for Ras-MAPKproliferation and PI3K-Akt-anti-apoptosis, could be involved in response to weight change by dietary calorie restriction and/or exercise training. Considering prevalence of obesity or overweight that becomes apparent over the world, understanding the underlying mechanisms among weight control, endocrine change and cancer risk is critically important. Future studies using "-omics" technologies will be warrant for a broader and deeper mechanistic information regarding cancer prevention by weight control.
\end{abstract}

Keywords: Weight Control; Dietary Calorie Restriction; Physical Activity; IGF-1, Leptin, glucocorticoids, Cancer Prevention.

\section{Introduction}

Obesity rate in the U.S is growing rapidly during the past 20 years (CDC, 2006). It has become a serious world wide problem which is associated with increased risk for several chronic diseases, including cancer, diabetes, and cardiovascular disease. Studies showed evidence for a positive association between overweight/adiposity and cancer risk in esophagus, pancreas, colon, rectum, endometrium, kidney, and postmenopausal breast cancer (WCRF/AICR, 2007). Weight control, therefore, has become an important strategy against cancer and/or other chronic diseases. Body weight control is carried out by the balance of negative energy, which is tightly associated with dietary calorie intake and/or physical activity (energy expenditure). A positive energy balance, via increased dietary intake and/or decreased energy expenditure, results in increased weight and fat mass, or adiposity. Negative energy balance via decreased calorie intake or increased expenditure in adult may help maintain body weight and thus benefit health status. 
Calorie restriction is referred to decrease of energy intake without malnutrition. In calorie restriction regimens, proteins and all the essential micronutrients such as vitamins and minerals are kept same. Only the total amount of energy from fat and carbohydrate is reduced usually at $20-40 \%$ of the ad libitum-fed controls. The cancer preventive effect of caloric restriction has been found for almost one hundred years. The first animal study was done as early as 1909 by Moreschi, who observed that tumors transplanted into underfed mice grew slower than those in ad libitum fed mice (Moreschi et al., 1909). In the 1940s, Tannenbaum and colleagues found that reduced food intake decreased tumor incidence in experimental animals (Tannenbaum et al., 1947). Later on, the preventive effect of calorie restriction on cancer is confirmed in various animal models such as primate and rodent or various organs including mammary gland, prostate, colon, and skin. Calorie restriction has been shown to be effective in both spontaneously occurring and chemically induced cancers. Calorie restriction is also able to lessen cancer in genetically engineered models, e.g., p53 knock out mice and APC ${ }^{\text {min }}$ mice (Hursting SD, 1997; Mai V et al., 2003). To data, calorie restriction is found to be the most potent and effective dietary intervention strategy for cancer prevention in animal models (Hursting SD. et al., 2003).

The health benefit of physical activity (exercise), on the other hand, has been known for many decades. Accumulated evidence both in human studies and animal models has shown that physical activity is helpful in decreasing cancer risk. The epidemiologic studies on the relationship between physical activity and cancer prevention as reviewed by Friedenreich and Orensterin (2002) suggested that the evidence of cancer prevention by physical activity be convincing for colon and breast cancer, probable for prostate cancer, and possible for endometrium and lung cancer, although some other types of cancers seemed less sufficient and conclusive. Colon cancer is studied most with respect to physical activity in animal models. It was found that physical activity, both by forced treadmill and voluntary wheel, was effective in reducing azoxymethane-induced colon carcinomas in rats (Reddy BS et al., 1988; Thorling EB et al., 1994). However, the results were not conclusive in APC ${ }^{\text {min }}$ mice (Basterfield et al., 2005). The effect of physical activity on breast cancer prevention in animal models was reviewed by Thompson et al. (2004), indicating physical activity might inhibit mammary carcinogenesis, but the effect was less reproducible compared to calorie restriction. Overall, the impact of physical activity on cancer prevention is positive, but not consistent or potent as calorie restriction approach.

Despite many studies have been conducted, no mechanism of weight control on cancer prevention has been well-established. Enhancement of DNA repair and diminution of oxidative damage to DNA, as well as reduction of oncogene expression have been postulated. Weight loss, via calorie restriction and/or exercise, has been found to reduce certain circulating growth factors and hormones, such as IGF-1, adipocytokins, glucocorticosteroids, which are critical in maintenance of cellular growth, proliferation, cell cycle, and apoptosis function. Reduction of these growth factors and inhibition of 
these factors-dependent biological processes by weight control may contribute to the overall anti-carcinogenesis.

\section{IGF-1: a key modulator for cell growth and anti-apoptosis}

\subsection{IGF-1 system: IGF-1, IGF-1 binding proteins, IGF-1 receptor and IGF signaling}

Insulin-like growth factors (IGF-1 and IGF-2) are 70-amino-acid polypeptides that have high sequence similarity to insulin. Both IGF-1 and IGF-2 have metabolic functions, and play important roles in cellular proliferation and differentiation. The major function of IGF-2 seems related to embryonic growth and early development (DeChiara et al., 1990), but IGF-1 is more important in post-natal growth. The synthesis of IGF-1 is mainly regulated by growth hormone in liver. IGF-1 is majority produced in the liver but also all the other cells.

The circulating levels of IGF-1 and their bioavailability are modulated by a family of IGF binding proteins (IGF-BPs), which have six homologies. IGF-BP3 is the most abundant in humans. It is found that about $90 \%$ of IGF-1 in the serum is binding to IGFBP3, a complex formed that is very large and can not transport out of bloodstream. Free IGF-1 or IGF-1 that binds to IGF-BP1 and IGF-BP2 are able to across the capillary endothelium and reach target tissues (Voskuil et al., 2005). IGF-BPs are degraded by proteases both in the tissues and in the circulation, through which IGF-1 is freed and interacts with IGF-1 receptors. Furthermore, IGF-BPs can also modulate the process of IGF-1 binding to receptors and the IGF signaling (Holly et al., 1993; Clemmons, 1993).

IGF-1 receptor (IGF-1R) is a member of receptor tyrosine kinase super family. IGF-1R binds IGF-1 with the highest affinity, while it can also bind IGF-2 and insulin (Ullrich et al., 1986). In addition to IGF-1R, insulin receptor (IR) and IGF-2 receptor (IGF-2R) are able to bind IGF-1, but with less affinity. Studies showed that insulin receptor and IGF-1 receptor could form heterohybrid (Soos et al., 1993; Pandini et al., 2002).

Binding of IGF-1 to the receptors will induce autophosoporylation and activation of downstream signal network, such as phosphatidylinositol-3-kinase (PI3K) pathway. The phosphorylation of PI3K results in activation of Akt. Activated Akt will then inhibit the activation of interleukin-1 $\beta$-converting enzyme (ICE)-like protease, therefore suppresses apoptosis. Binding of IGF-1 to its receptor is also found to activate other pathways, such as MAPK pathway. Activation of MAP kinase will lead to increase of cell proliferation (Raushan et al., 2006).

\subsection{IGF-1 and cancer}

The IGF-1 system has been found to be involved in human development and the maintenance of a normal function and homeostasis of the cell growth in the body. Abnormal function in increased IGF-1 levels leads to break down of normal cell homeostasis and function, which usually found in cancer development. Studies showed that the neoplasia process may due to the elevation of IGF-1 in the circulation and/or the 
increased sensitivity of IGF-1R to the hormone. Increased IGF-1 stimulates cell proliferation and inhibited apoptosis in various cancer cells. The relationship of IGF-1 and cancer and the potential corresponding mechanism have also been studied extensively in human subjects and animal models.

In the study of colon cancer, it was found that the gene expression of IGF-1 was elevated in colon carcinomas (Tricoli et al., 1986). Similar results were also found in the human breast and lung tumors (Yee et al., 1989). Later on epidemiological studies showed plasma IGF-1 levels were positively associated with higher risk of cancer, especially prostate and breast cancer. Chan et al. (1998) first demonstrated a link between circulating IGF-1 and prostate cancer risk using a nested case-control study. The study showed that plasma IGF-1 levels were positively associated with prostate cancer risk. Comparing to men in the lowest quartile of plasma IGF-1 levels, men in the highest quartile had a 4.3 folder higher risk of prostate cancer (Chan et al., 1998). In women, IGF-1 was found to be positively associated with pre-menopausal breast cancer rather than post-menopausal breast cancer (Renehan et al., 2004 lancet; Voskuil et al., 2005a; Canzian et al., 2006). The correlation between circulating IGF-1 levels and cancer risk was also found in colon cancer and bladder cancer (Giovannucci et al., 2001; Zhao et al., 2003). In addition to cancer risk, elevated plasma IGF-1 was associated with benign prostatic hyperplasia, proliferation of colorectal mucosa, and colorectal adenomas (Khosravi J et al., 2001; Chokkalingam AP et al., 2002; Cats A et al., 1996; ReneHan AG et al., 2001; Teramukai S et al., 2002). Overall, the reported studies support that relatively high circulating IGF-1 levels may have a causal role in cancer development.

IGF-1 and IGF-1 signaling are also found to play an important role in skin cancer. Rho et al. (1996) found that the mRNA level of IGF-1 and IGF-1 receptor in dermal and epidermal of mouse skin was significantly increased in the skin papillomas and carcinomas. In order to detect the potential role of IGF-1 signaling in the multistage mouse skin carcinogenesis, DiGiovanni lab developed transgenic mice HK1.IGF-1, in which IGF-1 is over expressed in epidermis driven by a human keratin 1 promoter (Bol DK et al., 1997). The authors found that HK1.IGF-1 transgenic mice were more sensitive to tumor promoters such as TPA, chrysarobin, okadaic acid, and benzyl peroxide after initiated by DMBA than wild type mice. Comparing to wild type animals which received the same dose of carcinogen treatment, transgenic mice developed tumors more rapidly and the number of tumors per mouse were dramatically increased (Bol DK et al., 1997; Wilker E et al., 1999). In addition, squamous papillomas and carcinomas were found to developed spontaneously in a similar transgenic mouse model BK5.IGF-1, which over expresses IGF-1 in the basal layer of skin epidermis (DiGiovanni et al., 2000). Activation of IGF-1 receptor, epidermal hyperplasia and increased labeling index were also observed in these mice. Not only in chemically induced skin carcinogenesis, an altered IGF system were also found to contribute to HaCaT keratinocyte UV susceptibility (Thumiger et al., 2005). The above data suggested that constitutive expression of IGF-1 and activation of IGF-1 receptor signaling pathways in basal epithelial cells lead to tumor promotion, in 
which IGF-1 played an important role in skin cancer development. More recently, it was found that PI3K/Akt pathway is important in IGF-1 mediated skin promotion (Wilker et al., 2005). Inhibition of PI3K activity significantly blocked epidermal proliferation, as well as skin tumor development in DMBA initiated IGF-1 transgenic mice (Wilker et al., 2005).

\subsection{IGF-1 as a mediator in cancer prevention by weight control}

As discussed above, the high IGF-1 levels seem associated with the risk of cancer development and lowering IGF-1 levels via weight control appears to be related to a decreased cancer incidence. Thus, manipulating plasma IGF-1 levels have been applied in cancer prevention strategies. In order to test this hypothesis, a mouse model that has a genetic deletion of liver IGF-1 gene was generated (Wu Y et al., 2003). In these mice, IGF-1 levels are $25 \%$ of that in none transgenic mice. Lowering circulating IGF-1 significantly delayed mammary gland tumor development by carcinogen DMBA or C3 (1)/SV40 large T- antigen induced carcinogenesis (Wu Y et al., 2003). Fibroblasts lacking IGF-1 receptor were found to be highly resistant to transformation by simian virus $40 \mathrm{~T}$ antigen (Pietrzkowski $\mathrm{Z}$ et al., 1992; Sell C et al., 1993). Moore et al. (2008) found that the activation of the Akt and mTOR signaling pathways by tumor promoter TPA were significantly reduced in IGF-1 deficient mice, resulting a blockage of epidermal response to tumor promotion. Kari et al. (1999) found that the functional disruption of IGF-1R markedly inhibited breast cancer metastasis in the nude mice by suppressing cellular adhesion, invasion, and metastasis of breast cancer cells to the lung, lymph nodes, and lymph vessels.

Reducing plasma IGF-1 by weight control has been investigated in a number of studies. Ruggeri et al. (1989) first reported that dietary calorie restriction decreased serum IGF-1 significantly at the first and third week after the experiment started in female SpragueDawley rats. Hursting et al. (1993) found serum IGF-1 in 40\% of calorie restricted rats was only $44 \%$ of ad libitum fed controls. The author also infused human recombinant IGF-1 back to the dietary restricted rats by using osmotic minipumps. Infusion of IGF-1 restored cell proliferation activity and enhanced mitogen responsiveness in dietary restriction treated rats (Hursting et al., 1993). In a tumor study of p53 deficient mice, $20 \%$ of calorie restriction decreased circulating IGF-1 by $26 \%$ and restoration of IGF-1 in calorie restricted mice did not change the tumor incidence significantly, but increased cell proliferation and inhibited apoptosis dramatically (Dunn et al., 1997). Study by Thompson also found that $40 \%$ of calorie restriction reduced circulating IGF-1 by half in rats and restoration IGF-1 failed to have effect on mammary tumor incidence (Zhu et al., 2005). Studies in our lab also found that IGF-1 was significantly decreased by dietary calorie restriction and restoration of IGF-1 significantly abolished PI3K reduction in treadmill exercised mice with limited feeding at same amount as sedentary control (Xie et al., 2007). Overall, the above results showed that reduction of IGF-1 levels and thus down-regulation of IGF-1 signaling pathways as a consequence of dietary restriction 
could contribute to anti-tumorigenesis. Restoration of IGF-1 abrogated, at least in part, the protective effect of calorie restriction on carcinogenesis.

The impact of physical activity on IGF-1 reduction and cancer prevention is complicated. As reviewed by Kaaks et al. (2004), physical activity decreased IGF-1 level in children and adolescents. But for adults, the plasma IGF-1 levels were not decreased either by short bout exercise or physical training. Studies showed that weight control by long term exercise could decrease IGF-1. For example, a recent published paper found that plasma concentrations of IGF-1 were significantly lower in endurance runners than sedative controls (Fontana et al., 2006). In animal model, our lab found that exercise alone with ad libitum feeding was not sufficient to decrease plasma IGF-1 levels. When the exercised mice was fed with a same amount as their sedentary counterpart, plasma levels of IGF-1 were modestly but significantly reduced (unpublished data). Nevertheless, the evidence by ours and others indicate a negative energy balance appears to be a fundamental requirement for IGF-1 reduction and potential cancer prevention.

\section{Adipocytokines: a linkage of adipose and cancer risk}

Adipocytokines are secretary products of adipose tissue and have metabolic and endocrine functions. They include leptin, adiponectin, resistin, and visfatin, etc., which have been identified and studied recently for a potential relationship between obesity and cancer risk (Korner et al., 2005).

\subsection{Leptin}

Leptin gene, which is also called obese (ob) gene, encodes a $16 \mathrm{kDa}$ protein (Zhang et al., 1994). As an adipocytokine, leptin is secreted mainly by adipose tissue. Other tissues, such as placenta, ovaries, skeletal muscle, pituitary gland, stomach, and liver, are also able to produce leptin. The major factor that affects circulating leptin levels is adipose tissue mass (Maffei et al., 1995). Increased body weight has been shown to be positively associated with high level of plasma leptin (Frederich RC et al., 1995). Leptin was found to regulate appetite and control body weight through affecting the hypothalamus, suppressing food intake and stimulating energy expenditure (Muoio et al., 2002). In addition to the central cirtuits, leptin also has effects in the periphery tissues, such as lung, intestine, skin, stomach, heart and other organs, though binding to leptin receptors (Margetic et al., 2002; Cornish et al., 2002).

Leptin receptors contain extracellular, transmembrane and intracellular domains. The extracellular domain is responsible for leptin binding and intracellular domain recruits and activates downstream substrates. Activation of leptin receptors was found to stimulate signaling pathways, such as JAK2/STAT3, Ras/ERK1/2 and PI3K/Akt/GSK3. Other signaling proteins induced by leptin were also found, including protein kinase $\mathrm{C}$, p38 kinase, and AP-1 component c-fos, c-jun, and junB, etc. (reviewed by Garofalo et al., 2006). 
Leptin is important in the regulation of energy balance. Obese (ob/ob) mice, which have leptin gene mutation, are found to be morbidly obese, infertile, hyperphagic, hypothermic, and diabetic (Huang et al., 2000). Infusion of recombinant leptin into these mice reduced food intake and decreased body weight (Campfield et al., 1995; Halaas et al., 1995). In diet induced obese mice, the circulating leptin was significantly elevated with the increase of body weight. Studies also showed these mice are resistant to peripherally administrated leptin (Van Heek et al., 1996). Compared to normal weight people, obese people usually developed hyperleptinemia and leptin resistant, which might due to a defect in transporting of leptin through the blood barrier (Banks et al., 1996; Caro et al., 1996).

Epidemiologic studies showed that moderately elevated serum leptin was associated with prostate cancer development (Stattin et al., 2001). People that have high leptin levels tend to have a large tumor (Chang et al., 2001, Saglam et al., 2003). However, some studies found there was no relationship between circulating leptin and prostate cancer risk (Lagiou et al., 1998; Stattin et al., 2003). In vitro, leptin is found to be a promoter in cancer cells. Studies showed that leptin induced cell proliferation in breast cancer ZR751 and HTB-26 cells via the activation MAPK and PI3K (Frankenberry et al., 2006). Leptin also simulated estrogen synthesis by increasing aromatase gene transcription and protein activity, which implied that leptin might be responsible for the resistance to antiestrogens during hormonal treatment of breast cancer (Sulkowska et al., 2006). In colon cancer cells, leptin induced cell growth and blocked apoptosis of human cancer HT29 cells via stimulation of ERK1/2 and NFאB pathway (Hardwick et al., 2001; Liu et al., 2001). In addition, the mitogentic activity of leptin has also been demonstrated in prostate, pancreatic, ovarian and lung cancer cells. Taken together, leptin seems to be important in tumor progression. Manipulation of plasma leptin might be effective in cancer prevention and treatment.

As discussed above, leptin was positively associated with body weight and body mess index. Weight control seems to be effective in lowering circulating leptin. Fontana et al (2006) showed that plasma concentrations of leptin were significantly lower in endurance runners than sedative controls. In animal models, it was found that $40 \%$ of calorie restriction significantly decreased the serum leptin levels in $\mathrm{APC}^{\mathrm{min}}$ mice compared to the control (Mai et al., 2003). Studies by our laboratory showed that the plasma level of leptin was significantly decreased in calorie restricted mice and exercised mice with paired feeding, but not in exercised mice with ad libitum feeding (Xie et al., 2007). Interestingly, we found that leptin in subcutaneous fat cells was not affected by weight control treatment (Xie et al., 2007). All the above evidence suggests that leptin be important in medicating the cancer protective effects of weight control. Further research is needed to characterize the specific role of leptin in cancer development. 


\subsection{Adiponectin}

Adiponectin is also an adipocytokine that is secreted in adipose tissue and plays an important role in obesity-related disorders. The gene of adiponectin is located on diabetes susceptibility locus chromosome 3q27 (Sonnenberg et al., 2004; Takahashi et al., 2000). Adiponectin was found to account for $0.01 \%$ of total plasma protein in human serum (Arita et al., 2002). It exists in several forms: trimers, hexamers, high molecular weight multimers (HMW), or globular form. HMW form was suspected to be the most bioactive form (Richards et al., 2006; Fisher et al., 2005).

Two adiponectin receptors have been identified. The signaling downstream of adiponectin receptors is still under investigation. Miyazaki et al. (2005) found that different forms of adiponectin have distinct biological effects, which may be through differential activation of downstream signaling.

Some evidence has showed that adiponectin is an insulin sensitizing hormone and may process anti-diabetic activities (Kantartzis et al., 2005). The blood level of adiponectin was found to be lower in obese people and was able to neutralization of LPS activity and anti-inflammation (Tsuchihashi et al., 2006; von et al., 2006). In addition, adiponectin was also found to be a modulator of lipid metabolism and might have preventive effect on cardiovascular disease. Kim et al. (2006) found that an increase of adiponectin concentrations or the maintenance of the higher levels was negatively associated with cardiovascular risk factors in nondiabetic CAD male patients, independent of adiposity and smoking status (Kim et al., 2006).

The potential anticancer properties of adiponectin have been investigated both in epidemiological study and animal models. There are three case control studies showed that low serum adiponectin levels were associated with an increase risk of breast cancer in women (Miyoshi et al., 2003b; Chen et al., 2005; Kang et al., 2005a). In breast cancer patient, people who have low serum adiponectin levels tended to have more aggressive tumor (Miyoshi et al., 2003a). The inverse relationship between serum adiponectin and endometrial cancer risk was also identified by two case control studies in Italy and Grace, respectively (Dal et al., 2004; Petridou et al., 2003). Adiponectin was found to be lower in prostate cancer patient comparing to healthy controls the levels were negatively correlated with histologic grade and disease stage (Goktas et al., 2005). Studies by Ishikawa et al. (2005) showed that in gastric cancer patients, their plasma adiponectin levels were significantly lower than healthy controls. In addition, the plasma adiponectin was negatively associated with tumor size, depth of invasion and tumor stage in undifferentiated gastric cancer (Ishikawa et al., 2005). A prospective nested case-control study conducted by Wei et al. (2005) observed that men with low plasma adiponectin levels had a higher risk of colon cancer than men with higher adiponectin. However, one study reported adiponectin was not associated with colorectal cancer (Lukanova et al., 2006). Overall, studies in human subjects provided some evidence that adiponectin could protect against certain type of cancer. 
The cancer preventive effect of adiponectin may partially explained by its ability in modulating the biology of tumor cells. Studies by Yokota et al., (2000) found that adiponectin suppressed the growth of myelocyte cells, induced apoptosis in myelotye leukemia cells, and inhibited TNF-alpha production. Adiponectin was found to inhibit breast cancer MDA-MB-231 and MCF-7 cells proliferation and induce cell cycle arrest and apoptosis in these cells (Kang et al., 2005b; Dieudonne et al., 2006). Bub et al., (2006) reported that adiponectin suppressed the growth of prostate cancer cells. In colon cancer cells, however, Ogunwobi et al. (2006) demonstrated that adiponectin was a promoter of colon cancer HT29 cells.

Studies showed that plasma adiponectin level was negatively associated with obesity, glucose and lipid levels, and insulin resistance (Bluher et al., 2006). Weight control through dietary calorie restriction and/or exercise seem to elevate plasma adiponectin, however, the results are not very conclusive. Studies showed calorie restricted rats had a high level of plasma adiponectin with reduced blood glucose, plasma insulin, and triglyceride levels when compared with ad libitum fed controls (Zhu et al., 2004). However, in a human study, the serum concentration of adiponection was not found to change in people after three weeks calorie restriction (Barnholt et al., 2005). For the effect of exercise, Jamurtas et al. (2006) showed that plasma adiponectin was not changed in people up to 48 hours post-acute exercis. Oberbach et al. (2006) reported that after four weeks of physical training, adiponectin levels was significantly increased in people who had type 2 diabetes. The changing of adiponectin levels was correlated with enhanced insulin sensitivity (Oberbach et al., 2006). Other adipocytokins, such as resistin and omentin, may also play a role in weight control-mediated cancer preventive effects, but their cellular and physiological function are still not clear (McTernan et al., 2006; Yang et al., 2006).

\section{Other hormones related to cancer prevention by weight control}

\subsection{Insulin}

Insulin is an important hormone that regulates blood glucose level. In the liver, it promotes glycogen synthesis by stimulating glycogen synthase and inhibiting glycogen phosphorylase. In muscle and fat tissues, insulin induces uptake of glucose via increasing GLUT4 expression. Insulin is also functioned as a moderate mitogen. After binding to its receptor, insulin may activate signaling pathways via phosphorylation of the insulinreceptor stubstarate-1, Akt, mitogen activated protein (MAP) kinase, and PI3K kinase (Rose et al., 1998; Finlayson et al., 2003). Therefore, insulin has been found to induce the growth of both normal and cancerous cells (Watkins L et al., Int. .Cancer 1990; Koenuma, M. et al., 1989; Bjork et al., 1993). Insulin also promotes the bioactivity of IGF-1 via either increasing the number of growth hormone receptors in the liver or reducing hepatic secretion of IGFBP1, which binds and inhibits the activity of IGF-1 (Underwood et al., 1994; Powell et al., 1991). 
Obesity or lack of physical activity is found to be a major factor inducing insulin resistance and further hyperinsulineamina. Epidemiological studies showed that increased plasma insulin was associated with a high risk of cancer (Kaaks R et al., 2000; Nilsen, TI et al., 2001). Dietary calorie restriction and/or regular exercise has been linked with a decreased plasma insulin in several studies (Zhu et al., 2005, Friedenreich et al. 2002, Giovannucci, E et al., 2001). It is noted that weight control via decreasing calorie intake or increasing energy expenditure can regulate glucose homeostasis and increase insulin sensitivity.

\subsection{Glucocorticoids}

Glucocorticoid hormones are a class of steroid hormones. The major function of these hormones are involved in regulation of glucose metabolism, such as stimulation of gluconeogenesis in the liver, inhibition of glucose uptake in the muscle and adipose tissue, stimulation of fat breakdown in adipose tissue, and mobilization of amino acids from extrahepatic tissues. Glucocorticoids are also important in fetal development and have anti-inflammatory and immunosuppressive effects.

Glucocorticoid hormones act though binding to intercellular glucocorticosteroid receptor. After binding with the hormone, the new formed receptor-ligand complex dissociates with heat shock proteins and then translocates into the nucleus, where it binds again to glucocorticoid response elements (GRE) and acts as a transcription factor. Glucocorticoid receptor usually works as a negative transcription factor, and it has been shown to inhibit the transcription of almost all immune system-related genes. In some cases, activated glucocorticoid receptor may interfere with other transcription factors, such as AP-1 and NFkB (Stöcklin et al., 1996; Subramaniam et al., 2003) that are crucial in the regulation of a number of genes involved in inflammation, differentiation, cell proliferation, apoptosis, oncogenesis, and other biological processes (Karin and Chang, 2001; Shaulian and Karin, 2001; Ghosh and Karin, 2002; Karin et al., 2002).

In addition, glucocorticoid steroids are potential tumor inhibitors. Administration of hydrocortisone in the diet showed preventive effect on the promoting phase of skin carcinogenesis in the mice (Trainin, 1963). There are a number of studies shown that glucocorticoid steroids are elevated in calorie restricted animals (Pashko and Schwartz, 1992; Yaktine et al., 1998). Adrenalectomy was found to decrease plasma corticosterone levels and abrogate the preventive effect of dietary restriction on skin tumor development (Pashko and Schwartz, 1992; Stewart et al., 2005). Similar results were also observed in lung carcinogenesis but not in mammary gland tumors (Pashko and Schwartz, 1996; Jiang et al., 2004). When administrating corticosterone in adrenalectomized mice, the cancer preventive effects of calorie restriction on skin carcinogenesis were restored as shown by our previous publication from the Birt lab (Stewart et al., 2005). Overall, the published data indicate glucocorticoids may be critical mediators in cancer prevention by calorie restriction.

\section{Molecular Targets of cancer prevention by weight control}




\subsection{Effects on cellular processes}

It is well known that cancer arises due to the loss of a normal growth control. In normal tissues, cell growth and cell death are highly regulated and balanced. In cancer, this regulation is disrupted, which is either from increased cell proliferation or loss of programmed cell death, or both.

The effect of calorie restriction on cell proliferation has been investigated in numerous studies. Lok et al. (1990) reported that $25 \%$ of calorie restriction decreased cell proliferation by $72 \%$ in mammary gland and $30-60 \%$ in skin, esophagus, bladder, and GI tract of female Swiss Webster mice. Pashko and Schwartz (1992) showed that 27\% of food restriction suppressed TPA-induced epidermal $\left[{ }^{3} \mathrm{H}\right]$-thymidine incorporation. In $\mathrm{C} 57 \mathrm{BL} / 6 \times \mathrm{C}_{3} \mathrm{HF}_{1}$ mice, a murine strain that develops liver tumor spontaneously, $40 \%$ of dietary restriction was found to decrease cell proliferation significantly in the liver (James et al, 1994). Dunn et al. (1997) demonstrated that $20 \%$ of calorie restriction significantly inhibited BrdU incorporation in the bladders of p53 knock out mice. Restoration of IGF-1 brought the cell proliferation back to the level of the control mice (Dunn et al., 1997). Comparing to ad libitum feeding, $30 \%$ of dietary calorie restriction significantly inhibited cell proliferation in carcinogen treated mouse skin (Fischer et al., 1998). Using a heavy water labeling, Hsieh et al. (2005) investigated a time-course of the effects of calorie restriction on cell proliferation rates in female $\mathrm{C} 57 \mathrm{BL} / 6 \mathrm{~J}$ mice. It showed the proliferation rates of mammary epithelial cells and $\mathrm{T}$ cells were markedly reduced within 2 weeks with calorie restriction regimen when compared to that of ad libitum fed mice. Two weeks after refeeding, the cell proliferation rates rebounded to the basal level (Hsieh et al., 2005). We found that the percentage of PCNA in skin epithelial cells was significantly lower in $20 \%$ of calorie restricted mice than ad libitum fed mice, as shown by immunohistochemistry staining (Xie et al., 2007). The percentage of the splenocyte in $\mathrm{S}$ phase was significantly reduced by $40 \%$ of calorie restriction in p53 knock out mice as well as wild type mice, as shown by Hursting et al. (1994). Studies in Thompson lab showed that cell cycle regulators, i.e., cyclin D1, cyclin E, cyclin-dependent kinase (CDK)-2, and CDK-4, were decreased by $40 \%$ of calorie restriction in rat mammal carcinomas, while cyclin-dependent kinase inhibitors (CKI), i.e., Kip1/p27 and Cip1/p21, increased (Zhu et al, 2003; Jiang et al, 2004). Overall, the effects of calorie restriction on cell proliferation are clear and reproducible in the animal models. For physical activity, studies in our lab found the cell proliferation rates of exercised mice with paired feeding had a lower rate than sedentary mice, but exercise with ad libitum feeding actually enhanced proliferative rates in epidermal cells, suggesting exercise alone without dietary calorie limitation might promote cellular proliferation and result in inconsistent impact on cancer protection (Xie et al., 2007).

Programmed cell death or apoptosis is highly regulated by a series of arranged morphological and biochemical events (Adams, 2003). It is important for maintenance of tissue homeostatsis, embryo development, and immune defense. Defects in apoptosis are thought to play an important role in cancer development (Gerl and Vaux, 2005; Lossi et 
al., 2005). Induction of apoptosis was observed in both normal liver and putative preneoplastic foci induced by hepatomitogen cyproerone acetate in dietary calorie restricted rats (Grasl-Kraupp et al, 1994). Dietary restriction was also found to induce apoptosis in the liver of $\mathrm{C} 57 \mathrm{BL} / 6 \times \mathrm{C}_{3} \mathrm{HF}_{1}$ mice (James et al, 1994). Increased apoptosis was observed in the bladder preneoplasia of $\mathrm{p}$-cresidine-treated $\mathrm{p} 53$-deficient mice by dietary calorie restriction (Dunn et al., 1997). In mammary gland, calorie restriction induced apoptosis in both premaglignant and malignant pathologies (Thompson et al., 2004). Thompson et al., reported that apoptosis regulatory molecules, i.e., Bcl-2, Bcl-xl, and XIAP, decreased and Bax and Apaf-1 increased significantly in the mammary carcinomas of calorie restricted rats when compared with that of the control rats (Thompson et al, 2004). They also reported that the activities of both caspases-9 and caspases- 3 were significantly induced and Akt phosphorylation was depressed by calorie restriction. The authors proposed that an induction of apoptosis by calorie restriction might be associated with its inhibitory effect on IGF-1 signaling. As for the physical activity, the research on apoptosis is sparse. Studies from our lab showed that caspase-3 activity but not caspase- 3 protein increased significantly in epidermis of dietary calorie restricted and treadmill exercised mice in comparison with the sedentary controls (Xie et al., 2007).

Collectively, all these data above indicate that modulation of cellular processes including inhibition of cell proliferation and restoration of apoptosis, is a molecular target in weight control for cancer prevention. Figure 1 shows a proposed mechanism by which weight control may inhibit cancer development via inhibiting the cross-talk between hormonedependent and TPA-promoted signaling pathways, resulting in modulating cellular

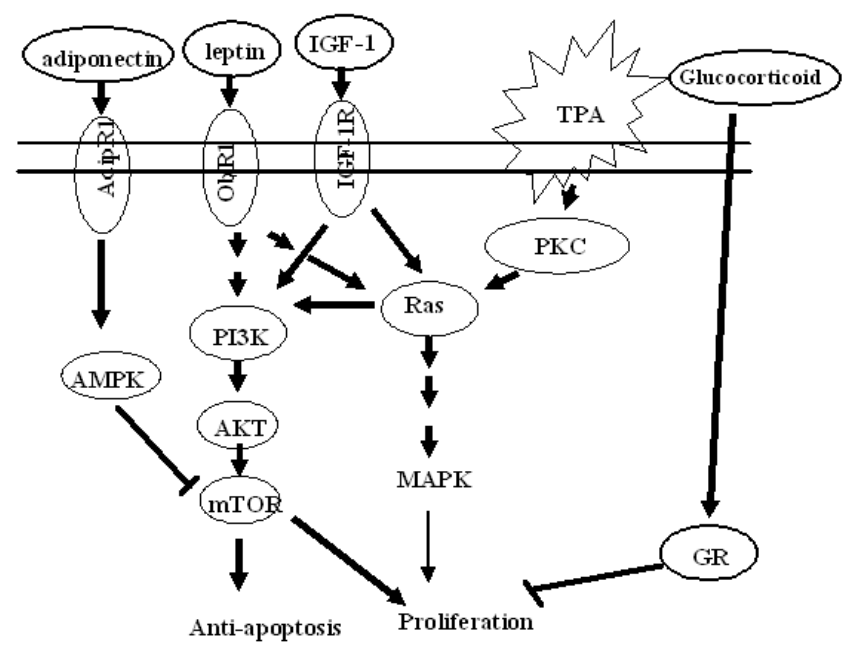

Fig. 1. A proposed mechanism by which weight control may inhibit cancer development via inhibiting the cross-talk between hormone-dependent and TPA-promoted signaling pathways, resulting in modulating cellular proliferation and anti-apoptosis. 
proliferation and anti-apoptosis. Weight loss reduces circulated growth factor and/or hormone levels such as IGF-1, leptin, adiponectin, and glucocorticoids, that thus inactivate TPA-induced signaling through hormone or growth factor-dependent cascades, e.g., Ras-MAPK and PI3K-Akt pathways. Finally, it may lead to an inhibition of TPAinduced cellular proliferation and elimination of IGF-1- persuaded anti-apoptosis.

\subsection{Reduction of oxidative stress}

Oxidative stress may injure cellular DNA, protein and lipids in the tissue. It is thus associated with ageing and many chronic diseases. In carcinogenesis, reactive oxygen and nitrogen species can attack DNA directly and induce DNA mutations. Oxidative stress also occurs by the reactive products of a peroxidation from various macromolecules, such as lipid peroxidation that may lead to protein and DNA modification. Cumulative evidence has been shown that long term calorie restriction in rodents extend maximum life span and decrease oxidative damage to DNA and proteins (reviewed by Richado Credilla et al., 2005). Qu et al. (2000) found that 60\% of calorie restriction completely abolished the increased oxidative damage in cloribrate-induced mouse liver (Qu et al., 2000)

\subsection{Other possible impact}

Calorie restriction may also interfere with the expression balance between oncogene and tumor suppressor gene directly. "Oncogenes refer to genes whose activation can contribute to the development of cancer" (Osborne et al., 2004). They are mutated versions from pro-oncogenes, which function in cell proliferation and differentiation. Over-expression of oncogenes usually causes out of control cellular growth. One of the most known oncogenes is Ras (Retrovirus-associated DNA sequences) family. Ras plays an important role in cell proliferation and can inactive tumor suppressors and promote cancer development (Fernandes et al, 1995). "Tumor suppressor genes refer to those genes whose loss of function results in the promotion of malignancy" (Osborne et al., 2004). Typically, a normal function of tumor suppressor genes is to inhibit cellular proliferation. Mutations of these genes usually result in a loss of their growth inhibition ability, which in turn may favors of cellular proliferation. Some examples of well known tumor suppressor genes include p53, retinoblastoma susceptibility gene, Wilms' tumors, neurofibromatosis type-1, and familial adenomatosis polyposis coli, etc. Previous studies have demonstrated that food restriction may induce an over-expression of tumor suppressor gene p53 (Fernandes $\mathrm{G}$ et al, 1995). In the Brown-Norway rats fed with calorie restricted diet or ad libitum diet, Hass et al. (1993) found that pancreatic acinar cells from calorie restricted animals had a lower growth rate and less N-methyl-N'-nitro$\mathrm{N}$-nitrosoguanidine (MNNG)-induced transformation. Calorie restriction derived cells showed decreased c-Ha-ras gene expression, lower rate of mutation of p53 tumor suppressor gene, and increased genomic methylations of DNA (Hass et al., 1993).

DNA repair occurs in the normal mammalian cells to repair DNA damage caused by multiple factors including oxidative stress. It reported that dietary calorie restriction 
enhanced DNA repair ability against DNA damage caused by UV exposure (Lipman et al, 1989; Weraarchakul et al, 1989). Hursting et al. (1999) suggested that both calorie restriction and exercise could induce DNA repair pathway, therefore block the early stage of carcinogenesis. However, 30\% of calorie restriction failed to activate DNA repair pathways and inhibit tumor development in the DNA mismatch deficient mice (Tsao et al, 2002).

\section{Summary}

As we know more about the protective mechanisms of weight control, it is becoming apparent that it is not only one single mechanism involved. Most likely, it is a combination of multiple factors and multiple signaling pathways involved. Hundreds of biological molecules may cooperate in this network complex. Therefore, traditional molecular biology techniques seem not to meet the requirement to gain a broader and deeper overview of the mechanisms. Fortunately, recently developed technologies named "-omics" may provide us a chance to take a global view of these biological processes. The "-omics" techniques such as genomics, proteomics, and lipidomics, etc, usually present a profile change of gene, protein, and lipid expression, respectively. Microarray study is the first step to obtain a gene expression profile, together with proteomics and lipidomics may generate a clear picture of a profiling response to the weight loss treatment. Our on-going studies by using these state of the art technologies hopefully lead to a better and deeper mechanistic information regarding the established cancer prevention by weight control in the near future.

\section{Acknowledgments}

This work was supported by an Innovative Research Grant from the Terry C. Johnson Center for Basic Cancer Research, Kansas State University, NIH COBRE Award P20 RR15563 and Kansas State matching support, and NIH R01 CA106397. This is a journal contribution \#09-017-J by the Kansas Agricultural Experiment Station, Kansas State University.

\section{References}

J. M. Adams, Genes Dev 17, 2481 (2003).

Y. Arita, S. Kihara, N. Ouchi, K. Maeda, et al., Circulation 105, 2893 (2002).

W. A Banks, A. J. Kastin, W Huang, J. B. Jaspan, and L. M. Maness, Peptides 17, 305 (1996).

K. E. Barnholt, A. R. Hoffman, P. B. Rock, S. R. Muza, et al., Am J Physiol Endocrinol Metab 290, E1078 (2006).

L. Basterfield,L, J. M. Reul, and J. C. Mathers, J Nutr. 135, 3002S (2005).

J. Björk, J. Nilsson, R. Hultcrantz, and C. Johansson,. Scand J Gastroenterol 28, 879 (1993).

M. Blüher, J. W. Jr Bullen, J. H. Lee, S. Kralisch, et al., J Clin Endocrinol Metab 91, 2310 (2006).

D. K. Bol, K. Kiguchi, I. Gimenez-Conti, T. Rupp, and J. DiGiovanni, Oncogene 14, 1725 (1997). 
J. D. Bub, T. Miyazaki, and Y. Iwamoto, Biochem Biophys Res Commun. 340, 1158 (2006).

L. A. Campfield, F. J. Smith, Y. Guisez, R. Devos, and P. Burn, Science 269, 546 (1995).

F. Canzian, J. D. McKay, R. J. Cleveland, L. Dossus et al., Br J Cancer. 94, 299 (2006).

J. F. Caro, J. W. Kolaczynski, M. R. Nyce, Ohannesian JP et al., Lancet 348, 159 (1996).

A. Cats A, R. P. Dullaart, J. H. Kleibeuker, F. Kuipers et al., Cancer Res. 56, 523 (1996).

CDC, MMWR Morb Mortal Wkly Rep. 55, 985 (2006).

J.M. Chan, M. J. Stampfer, E. Giovannucci E, P. H. Gann et al., Science. 279, 563 (1998).

S. Chang, S.D. Hursting, J. H. Contois JH, S. S. Strom et al., Prostate. 46, 62 (2001).

D. C. Chen, Y. F. Chung, Y. T. Yeh, H. C. Chaung et al., Cancer Lett. 237, 109 (2006).

A. P. Chokkalingam, Y. T. Gao, J. Deng J, F. Z. Stanczyk et al., Prostate. 52, 98 (2002).

D. R. Clemmons, Mol. Reprod. Dev. 35, 368 (1993).

J. Cornish, K. E. Callon, U. Bava, C. Lin et al., J Endocrinol. 175, 405 (2002).

M. L. Dal, L. S. Augustin, A. Karalis, R. Talamini et al., J Clin Endocrinol. Metab 89, 1160 (2004).

T. M. DeChiara, A. Efstratiadis, and E. J.Robertson, Nature 345, 78 (1990).

M. N. Dieudonne, M. Bussiere, S. E. Dos, M. C. Leneveu et al., Biochem. Biophys. Res Commun. 345, 271 (2006).

J. DiGiovanni, D. K. Bol, E. Wilker, L. Beltran et al., Cancer Res 60, 1561(2000).

S. E. Dunn, F. W. Kari, J. French, J. R. Leininger et al., Cancer Res. 57, 4667 (1997).

G. Fernandes, B. Chandrasekar, D. A. Troyer, J. T. Venkatraman et al., Proc Natl Acad Sci U S A. 92, 6494 (1995).

C. A. Finlayson, J. Chappell, J. W. Leitner, M. L. Goalstone, et al., Metabolism 52, 1606 (2003).

F. F. Fisher, M. E. Trujillo, W. Hanif, A. H. Barnett et al., Diabetologia 48, 1084 (2005).

L. Fontana, S. Klein and J. O. Holloszy, Am J Clin Nutr. 84, 1456 (2006).

K. A. Frankenberry, H. Skinner, P. Somasundar, D. W. McFadden et al., Int J Oncol. 28, 985 (2006).

R. C. Frederich, A. Hamann, S. Anderson, B. Löllmann et al., Nat Med. 1, 1311 (1995).

C. M. Friedenreich and M. R. Orenstein, J Nutr. 132, 3456S (2002).

C. Garofalo and E. Surmacz, J Cell Physiol. 207, 12(2006).

R. Gerl and D.L. Vaux Carcinogenesis. 26, 263 (2005).

S. Ghosh and M. Karin, cell 109, S81 (2002).

E. Giovannucci, J Nutr 131, 3109S (2001).

S. Goktas, M. I. Yilmaz, K. Caglar, A. Sonmez et al., Urology 65, 1168 (2005).

B. Grasl-Kraupp, W. Bursch, B. Ruttkay-Nedecky, A. Wagner et al., Proc Natl Acad Sci U S A. 91, 9995 (1994). 
16 Jiang \& Wang

J. L. Halaas, K. S. Gajiwala, M. Maffei, S. L. Cohen et al., Science. 269, 543 (1995).

J. C. Hardwick, G.R. Van Den Brink, G. J. Offerhaus GJ, S. J. Van Deventer et al., Gastroenterology. 121, 79 (2001)

B. S. Hass, R. W. Hart, M. H. Lu, and B. D. Lyn-Cook, Mutat. Res. 295, 281 (1993).

J. M. Holly, D.C. Claffey, S. C. Cwyfan-Hughes, V. J. Frost et al., Growth Regul. 3, 88 (1993).

E. A. Hsieh, C. M. Chai, and M. K. Hellerstein, Am J Physiol Endocrinol Metab. 288, E965 (2005).

L Huang and C. Li, Cell Res. 10, 81 (2000).

S. D. Hursting and F. W. Kari, Mutat Res. 443, 235 (1999).

S. D. Hursting, J.A. Lavigne, D. Berrigan, and S. N. Perkins, Annu Rev Med 54, 131 (2003).

S. D. Hursting, S. N. Perkins, C. C. Brown, D. C. Haines et al., Cancer Res. 57, 2843 (1997).

S. D. Hursting, B.R. Switzer, J. E. French, and F. W. Kari, Cancer Res. 53, 2750 (1993).

M. Ishikawa, J. Kitayama, S. Kazama, T. Hiramatsu et al., Clin Cancer Res. 11, 466 (2005).

S. J. James and L. Muskhelishvili Cancer Res. 54, 5508 (1994).

A. Z. Jamurtas, V. Theocharis V, Koukoulis G, Stakias N et al., Eur J Appl Physiol. 97, 122 (2006).

W. Jiang, Z. Zhu, J. N. McGinley, and H. J. Thompson J Nutr. 134, 1152 (2004).

R. Kaaks, Novartis Found Symp.262, 247 (2004).

J. H. Kang, Y. Y. Lee, B. Y. Yu, B.S. Yang et al., Arch. Pharm. Res 28, 1263 (2005).

K. Kantartzis, A. Fritsche, O. Tschritter, C. Thamer et al., Obes. Res 13, 1683 (2005).

F. W. Kari, S. E. Dunn, J. E. French, and J. C. Barrett, J Nutr. Health Aging 3, 92 (1999).

M. Karin, Y. Cao, F. R. Greten, Z. W. Li et al., Nat Rev Cancer.2 301 (2002).

M. Karin and L. Chang. J Endocrinol. 169, 447 (2001).

J. Khosravi, A. Diamandi, J. Mistry, and A. Scorilas, J Clin Endocrinol Metab. 86, 694 (2001).

O. Y. Kim, S. J. Koh, Y. Jang, J. S. Chae et al., Clin Chim Acta. 370, 63 (2006).

M. Koenuma, T. Yamori, and T. Tsuruo, Jpn J Cancer Res 80, 51 (1989).

R. T. Kurmasheva and P. J. Houghton, Biochim Biophys Acta. 1766, 1 (2006).

P. Lagiou, L. B. Signorello, D. Trichopoulos, A. Tzonou et al., Int J Cancer. 76, 25 (1998).

J. M. Lipman, A. Turturro, and R.W. Hart, Mech Ageing Dev. 48, 135 (1989).

Z. Liu, T. Uesaka, H. Watanabe, and N. Kato, Int J Oncol. 19, 1009 (2001).

E. Lok, F. W. Scott, R. Mongeau, E. A. Nera, et al., Cancer Lett. 51, 67 (1990).

A. Lukanova, S. Soderberg, R. Kaaks, E. Jellum et al., Cancer Epidemiol Biomarkers Prev 15, 401 (2006).

M. Maffei, J. Halaas, E. Ravussin, R. E. Pratley et al., Nat Med. 1, 1155 (1995).

V. Mai, L. H. Colbert, D. Berrigan, and Perkins SN, Cancer Res. 63, 1752 (2003). 
S. Margetic, C. Gazzola, G. G. Pegg, and R. A. Hill, Int J Obes Relat Metab Disord. 26, 1407 (2002).

P. G. McTernan, C. M. Kusminski, and S. Kumar, Curr. Opin. Lipidol. 17, 170 (2006).

T. Miyazaki, J. D. Bub, M. Uzuki, Y. Iwamoto et al., Biochem. Biophys. Res Commun. 333, 79 (2005).

Y. Miyoshi, T. Funahashi, S. Kihara, T. Taguchi et al., Clin Cancer Res 9, 5699 (2003).

T. Moore, S. Carbajal, L. Beltran, S.N. Perkins et al., Cancer Res. 68, 3680 (2008).

Moreschi C. Z. Immunitaetsforsch. 2, 651 (1909).

D. M. Muoio and G. Lynis Dohm, Best Pract Res Clin Endocrinol Metab. 16, 653 (2002).

T. I. Nilsen and L. J. Vatten, Br J Cancer. 84, 417 (2001).

A. Oberbach, A. Tonjes, N. Kloting, M. Fasshauer et al., Eur. J Endocrinol. 154, 577 (2006).

O. O. Ogunwobi and I. L. Beales, Regul Pept. 134, 105 (2006).

C. Osborne, P. Wilson and D. Tripathy, Oncologist. 9, 361 (2004).

G. Pandini, F. Frasca, R. Mineo, L. Sciacca et al., IJ Biol. Chem. 277, 39684 (2002).

L. L. Pashko and A. G. Schwartz, Carcinogenesis 17, 209 (1996).

L. L. Pashko and A. G. Schwartz, Carcinogenesis 13, 1925 (1992).

E. Petridou, C. Mantzoros, N. Dessypris, P. Koukoulomatis et al., J Clin Endocrinol. Metab 88, 993 (2003).

Z. Pietrzkowski, C. Sell, R. Lammers, A. Ullrich et al., Mol Cell Biol. 12, 3883 (1992)

D. R. Powell, A. Suwanichkul, M. L. Cubbage, L.A. DePaolis et al., J Biol Chem. 266, 18868 (1991).

B. Qu, B. Halliwell, C. N. Ong, B. L. Lee et al., FEBS Lett. 473, 85 (2000).

B. S. Reddy, S. Sugie, and A. Lowenfels Cancer Res. 48, 7079 (1988).

A. G. Renehan, M. Zwahlen, C. Minder, S. T. O'Dwyer et al., Lancet. 63, 1346 (2004).

O. Rho, D. K. Bol, J. You, L. Beltran et al., Mol. Carcinog. 17, 62 (1996).

A. A. Richards, T. Stephens, H. K. Charlton, A. Jones et al., Mol Endocrinol. 20, 1673 (2006).

D. W. Rose, S. Xiao, T. S. Pillay, W. Kolch et al., Oncogene 17, 889 (1998).

B. A. Ruggeri, D. M. Klurfeld, D. Kritchevsky, and R. W. Furlanetto, Cancer Res. 49, 4130 (1989).

K. Saglam, E. Aydur, M. Yilmaz and S. Göktaş, J Urol. 169, 1308 (2003).

C. Sell, M. Rubini, R. Rubin, J. P. Liu, A. Efstratiadis, Proc Natl Acad Sci U S A. 90, 11217 (1993).

E. Shaulian and M. Karin, Oncogene. 20, 2390 (2001).

Sonnenberg,G.E., Krakower,G.R., and Kissebah,A.H Obes. Res 12, 180 (2004).

Soos,M.A., Field,C.E., and Siddle,K. (1993). Biochem. J 290, 419 (1993). 
18 Jiang \& Wang

P. Stattin, R. Kaaks, R. Johansson, R. Gislefoss et al., Cancer Epidemiol Biomarkers Prev. 12, 474 (2003).

P. Stattin, S. Söderberg, G. Hallmans, A. Bylund et al., J Clin Endocrinol Metab. 86, 1341 (2001).

J. W. Stewart, K. Koehler, W. Jackson, J. Hawley et al., Carcinogenesis. 26, 1077 (2005).

E. Stöcklin, M. Wissler, F. Gouilleux, and B. Groner, Nature. 383, 726 (1996).

N. Subramaniam, J. Campión, I. Rafter, and S. Okret, Biochem J. 370, 1087 (2003).

Sulkowska M, Golaszewska J, Wincewicz A, Koda M, Baltaziak M, Sulkowski S. Pathol Oncol Res. 12, 69 (2006).

M. Takahashi, Y. Arita, K. Yamagata, Y. Matsukawa et al., Int. J Obes. Relat Metab Disord. 24, 861 (2000).

Tannenbaum A, Ann. NY Acad. Sci. 49, 5 (1947).

S. Teramukai, T. Rohan, K. Y. Lee, H. Eguchi et al., Jpn J Cancer Res. 93, 1187 (2002)

H, J, Thompson, Z. Zhu, W. Jiang, (a) Cancer Res. 64, 1541 (2004)

H. J. Thompson, Z, Zhu, W. Jiang, (b) J. Nutr. 134, 3407S (2004)

E. B. Thorling, N. O. Jacobsen, and K. Overvad, Eur J Cancer Prev. 2:77-82.(1993)

S. P. Thumiger, T. E. Adams, G. A. Werther, C. J.Wraight, et al., Growth Factors 23, 151 (2005).

Trainin N., Cancer Res. 23, 415 (1963).

J. V. Tricoli, L. B. Rall, C. P. Karakousis, L. Herrera et al., Cancer Res 46, 6169 (1986).

J. L. Tsao, S. Dudley, B. Kwok, A. E. Nickel et al., Carcinogenesis. 23, 1807 (2002).

H. Tsuchihashi, H, Yamamoto, K. Maeda, S. Ugi et al., J Surg Res. 134, 348 (2006).

A. Ullrich, A. Gray, A. W. Tam, T. Yang-Feng, et al., EMBO J 5, 2503 (1986).

L. E. Underwood, J. P. Thissen, S. Lemozy et al., Horm Res. 42, 145 (1994).

M. Van Heek, D. E. Mullins, M. A. Wirth, M. P. Graziano et al., Horm Metab Res. 28, 653 (1996).

M. von Eynatten, A. Hamann, D. Twardella, P. P. Nawroth et al., Clin Chem 52, 853 (2006).

D. W. Voskuil, A. Vrieling, L. J. van't Veer, E. Kampman et al., Cancer Epidemiol Biomarkers Prev 14, 195 (2005).

L. F. Watkins, L. R. Lewis, and A. E. Levine, Int J Cancer. 45372 (1990).

WCRF/AICR, http://www.dietandcancerreport.org/?p=ER

E. K. Wei, E. Giovannucci, C. S. Fuchs, W. C. Willett et al., J Natl Cancer Inst 97, 1688 (2005).

N. Weraarchakul, R. Strong, W. G. Wood, and A. Richardson, Exp Cell Res. 181, 197 (1989)

E. Wilker, D. Bol, K. Kiguchi, T. Rupp, et al., Mol Carcinog. 25, 122 (1999).

E. Wilker, J. Lu, O. Rho, S. Carbajal, et al., Mol. Carcinog. 44, 137 (2005).

Y.Wu, K. Cui K, K. Miyoshi K, L. Hennighausen et al., Cancer Res. 63, 4384 (2003).

L. Xie, Y. Jiang, P. Ouyang, J. Chen, et al., J Biol Chem. 282, 28025 (2007). 
A. L. Yaktine, R. Vaughn, D. Blackwood, E. Duysen and D.F. Birt, Mol Carcinog. 21, 62 (1998).

R. Z. Yang, M. J. Lee, H. Hu, J. Pray et al., Am J Physiol Endocrinol Metab. 290, E1253 (2006).

D. Yee, S. Paik, G. S. Lebovic, R. R. Marcus et al., Mol Endocrinol. 3, 509 (1989).

T. Yokota, K. Oritani, I.Takahashi, J. Ishikawa et al., Blood 96, 1723 (2000).

Y. Zhang, R. Proenca, M. Maffei, M. Barone et al., Nature 372, 425 (1994).

H. Zhao, H. B. Grossman, M. R. Spitz, S. P. Lerner et al., J Urol. 169, 714(2003).

M. Zhu, J Miura, L. X. Lu, M. Bernier et al., Exp Gerontol. 39, 1049 (2004).

Z. Zhu Z, W. Jiang, J. McGinley, P. Wolfe, and H. J. Thompson, Mol Carcinog. 42(3):170-6 (2005). 